\title{
HALANAY INEQUALITY, YORKE 3/2 STABILITY CRITERION, AND DIFFERENTIAL EQUATIONS WITH MAXIMA
}

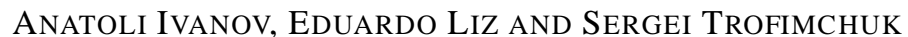

(Received June 19, 2000, revised January 29, 2001)

\begin{abstract}
We present an extension of the well-known 3/2-stability criterion by Yorke for two term functional differential equations. We prove the exact nature of the obtained stability region which coincides with the Yorke result in the special case when the decay term is absent. Moreover, we reveal some interesting links existing between the Yorke conditions, Halanay inequalities and differential equations with maxima, all of them essentially involving the maximum functionals.
\end{abstract}

1. Introduction. It can be observed that several important approaches in the stability theory of delay differential equations of the form

$$
x^{\prime}(t)+a x(t)+b f\left(t, x_{t}\right)=0, \quad t \in \boldsymbol{R}, \quad\left(x_{t}(s)=x(t+s), s \in[-h, 0]\right),
$$

involve the maximum functional $\max _{s \in[-h, 0]} \phi(s)$ on the space $C:=C([-h, 0], \boldsymbol{R})$ in an essential and subtle way which sometimes is far away from the simple use of the sup-norm relations like

$$
\left|f\left(t, x_{t}\right)\right| \leq\left\|x_{t}\right\|_{C}=\max \left|x_{t}\right| .
$$

To be more specific, we mention the Barnea's method [2] and the following two remarkable advances of the late sixties. The first one is the Halanay inequality and its various applications (see [7] and Section 3 of the present paper), and the second one is a version by Yorke of the $3 / 2$-stability criterion (see [24] and Section 2 below). For example, the $3 / 2$ criterion says that if $f: \boldsymbol{R} \times C \rightarrow \boldsymbol{R}$ is continuous, $a=0,0<b h<3 / 2$, and the following Yorke condition

$$
\min \phi=-\max (-\phi) \leq f(t, \phi) \leq \max \phi
$$

is satisfied, then the trivial solution of (1.1) is uniformly asymptotically stable. Moreover, the constant $3 / 2$ gives the exact upper bound for $b h$. Notice that the bifurcation character of this number was already indicated by Myshkis [10] for linear delay differential equations. An important contribution by Yorke was in the extension of the Myshkis criterion to nonlinear systems for the nonlinearities that can be appropriately majorized by maximum functionals.

2000 Mathematics Subject Classification. 34K20.

Key words and phrases. Functional differential equations, asymptotical stability, 3/2-type stability conditions.

A. Ivanov was supported in part by the Australian Research Council.

E. Liz was supported in part by D.G.E.S.I.C (Spain), project PB97-0552.

S. Trofimchuk was supported in part by FONDECYT (Chile), project 8990013 and by the Australian Research Council. 
On the other hand, by applying to (1.1) the method of the Halanay inequality

$$
x^{\prime}(t)+a x(t)+b \max x_{t} \leq 0,
$$

with its various generalizations, one can even more relax the Yorke condition (1.3), requiring only (1.2) together with the following additional assumption introduced in [14]:

$$
|f(t, \phi)-\phi(0)| \leq h \max \left|\phi^{\prime}\right| \quad \text { for any } t \in \boldsymbol{R} \quad \text { and } \quad \phi \in C^{1}[-h, 0] .
$$

As a result, the global exponential stability of the trivial solution always follows.

The Halanay and Yorke theorems were subjects of a number of subsequent studies and improvements, so that it would be impossible to give here a complete list of corresponding references. We list only several most relevant papers $[5,6,9,11,13,15,22,23]$ (some additional relevant references can be found in $[3,8])$ together with recent papers $[14,16,19]$ by the authors.

Both results mentioned above provide a strong indication that it is the differential equations with "maxima" $(f(t, \phi)=\max \phi)$ rather than the linear ones $(f(t, \phi)=\phi(-h))$ that represent natural comparison systems in the stability theory of quasilinear functional differential equations. Let us demonstrate this idea by using the simple equation

$$
x^{\prime}=-b \max _{s \in[t-1, t]} x(s),
$$

which can be viewed as a comparison equation for (1.1), (1.3) with $a=0$. By the Yorke theorem, this equation is uniformly asymptotically stable if $0<b<3 / 2$. This fact by itself does not have much interest, since one can easily prove (see [16]) that the above equation is actually asymptotically stable for all $b>0$. In Section 4 we point out a strong connection between the number $3 / 2$ and the nonhomogeneous $T$-periodic differential equation

$$
x^{\prime}=-b \max _{s \in[t-1, t]} x(s)+f(t) .
$$

In fact, a family of variational equations can be associated with the periodic problem (1.6), and the number $b=3 / 2$ is determined completely by the spectrum of this family. In particular, we will show that (1.6) has a globally exponentially stable $T$-periodic solution for every $T$-periodic forcing term $f(t)$ if and only if $b \in(0,3 / 2)$. We notice again that for the corresponding linear equation

$$
x^{\prime}=-b x(t-1)+f(t),
$$

the number $3 / 2$ is not basic at all (the equation is asymptotically stable for $0<b<\pi / 2$ ). Our paper reveals, for the first time we believe, this new aspect of the theory of equations with maxima (see Section 4 for details and more references about this type of delay systems). Moreover, now we are in a position to deduce a whole family of exact Yorke type stability criteria for more general systems (see Theorems 2.9 and 2.10 below).

In the present paper we establish various sufficient conditions for the global exponential stability of the zero solution for equations like (1.1). Moreover, we present explicit convergence estimates in the case of such stability. We point out a sharp and sometimes exact nature of the obtained results. Finally, a basic feature of the paper is in a special emphasis we put 
on the links existing between the following three objects: the Yorke type equations (1.1), (1.3), the Halanay inequality (1.4) with its generalizations, and the differential equations with maxima

$$
x^{\prime}(t)+a x(t)+b \max x_{t}=f(t) .
$$

The paper is organized as follows. In Section 2 we present our main stability results (Theorems 2.4 and 2.6 with corollaries), whose proof can be found in Sections 5-7. Section 3 deals with the Halanay-type inequalities, while Section 4 is devoted to equations with maxima of the form (1.7). The results of the third and fourth Sections are of crucial importance in the proof of Theorems 2.9 and 2.10.

2. Stability criteria. The main results of this section concern questions like the following one: for what values of the nonnegative parameters $a=\|a(t)\|_{\infty}$ and $b=\|b(t)\|_{\infty}$ the functional differential equation

$$
x^{\prime}(t)+a(t) x(t)+b(t) x(t-h)=0, \quad t, x \in \boldsymbol{R},
$$

with nonnegative coefficient $a(t)$ is uniformly asymptotically stable? In some cases it is possible to get a satisfactory answer by comparing the nonautonomous equation with the corresponding linear autonomous equation obtained by replacing the coefficients by their limiting values. This is precisely the case when $b(t) \leq 0$, as the following result proved in [4] shows:

Proposition 2.1. Assume that (2.1) with bounded $a, b \in C(\boldsymbol{R}, \boldsymbol{R}), b(t) \leq 0$ is uniformly asymptotically stable. Then, for any bounded continuous $c(t)$ such that $|c(t)| \leq$ $-b(t)$, the equation $x^{\prime}(t)+a(t) x(t)+c(t) x(t-h)=0$ is also uniformly asymptotically stable.

When $b(t) \geq 0$, this comparison principle does not apply. This fact was first pointed out by A. Myshkis for $a=0, h=1$. He also showed that the "limit" value of $\|b(t)\|_{\infty}$ is $3 / 2$, which is less than $\pi / 2$ suggested by the analysis of the corresponding autonomous equation (see $[8,10])$. Therefore, in general, the analog of Proposition 2.1 is not valid for the case $b(t) \geq c(t) \geq 0$. A different approach is needed, and we present it below. Our methods allow us to describe the exact stability domains in the parameter space in several important cases.

Our main results concern the stability of the functional differential equation

$$
\text { (2.2) } x^{\prime}(t)+g(t, x(t))+b(t) f\left(t, x_{t}\right)=0, \quad t \in \boldsymbol{R}, \quad\left(x_{t}(s)=x(t+s), s \in[-h, 0]\right),
$$

where $x_{t} \in C=C[-h, 0]$ with $h>0$ and $b \in L^{\infty}\left(\boldsymbol{R}, \boldsymbol{R}_{+}\right)$. In many situations modeled by (2.2), the coefficient $b(t)$ subject to $0 \leq b(t) \leq b$ can be viewed, for instance, as a control implied by an external observer, while the internal structure of the system is described by some 'hidden' continuous functionals $g(t, x), f(t, \varphi)$.

We will also assume that only the upper bound $b=\operatorname{ess}^{\sup _{t \in \boldsymbol{R}}} b(t)$ is known and that the continuous and 'almost linear' functional $f(t, \varphi)$ satisfies the Yorke condition (1.3). Regarding the nonlinearity $g(t, x)$, we will consider the following sequence of hypotheses:

(a) $x \cdot g(t, x)>0$ for all $x \neq 0$; 
(b) $g: \boldsymbol{R}^{2} \rightarrow \boldsymbol{R}$ is a Carathéodory function, and the equation $x^{\prime}+g(t, x)=h(t)$ has a unique (local) solution for every initial value problem and for every locally integrable $h(t)$;

(c) $\int_{t_{0}}^{+\infty} b(s) d s=\infty$ or $\int_{t_{0}}^{+\infty}|g(s, h(s))| d s=\infty$ for each $t_{0}$ and for every continuous function $h:\left[t_{0},+\infty\right) \rightarrow \boldsymbol{R}$ such that $\inf _{t \geq t_{0}}|h(t)|>0$.

We will also use the following condition implying (c):

(d) There exist $\mu>0$ and $q \in(0,1)$ such that

$$
\inf _{t \in \boldsymbol{R}} \int_{t}^{t+\mu}\left[M^{-1} g(s, h(s))+q b(s)\right] d s>1-q
$$

for every $M>0$ and for every continuous function $h: \boldsymbol{R} \rightarrow \boldsymbol{R}$ such that $h(t) \geq q M$. The same condition holds if we take $g_{1}(t, x)=-g(t,-x)$ instead of $g(t, x)$.

REMARK 2.2. It should be noted that (d) is satisfied if $\inf _{t \in \boldsymbol{R}} \int_{t}^{t+\mu} b(s) d s>0$ for some $\mu>0$ (see a dynamical interpretation of this inequality in [19]). Also, within our approach, conditions like ess $\sup _{t \in \boldsymbol{R}} h^{-1} \int_{t}^{t+h} b(s) d s \leq b$ can be considered in place of ess $\sup _{t \in \boldsymbol{R}} b(t) \leq b$.

Before presenting the last two hypotheses, we have to introduce several notations. Let $b=\|b\|_{\infty} \geq h^{-1}$ and $\zeta \in \boldsymbol{R}$ be fixed. Then, by (a), for the solution $x(t)$ of

$$
x^{\prime}(t)=-g(t, x(t))-b x(t-h), t \geq \zeta, \quad \text { and } \quad x(s)=-M<0, \quad s \leq \zeta,
$$

we can find $\alpha \in[\zeta, \zeta+h]$ such that i) $x(t)<0$ on $[\zeta, \alpha)$; ii) $x(\alpha)=0, x^{\prime}(\alpha)=b M>0$; iii) $x(t)>0$ for all $t \in I=(\alpha, \beta) \subseteq(\alpha, \alpha+h)$, where $\beta$ is assumed to be the maximal number having this property. Define $r_{0}(\zeta, M)=\max _{t \in I} x(t)$. Next, since the function $g_{1}(t, x)=$ $-g(t,-x)$ retains all stated above properties of $g(t, x)$, we can find the corresponding function $r_{1}(\zeta, M)$ in an analogous way. Finally, set $r(\zeta, M)=\max \left\{r_{0}(\zeta, M), r_{1}(\zeta, M)\right\}$.

Now, we are able to state our last two assumptions:

(e) $b h \leq q<1$ or $b h \geq 1$ and $M^{-1} r(\zeta, M) \leq q<1$ for some $q$ and all $M>0, \zeta \in \boldsymbol{R}$.

(f) $b h \leq 1$ or $b h>1$ and $M^{-1} r(\zeta, M) \leq 1$ for all $M>0, \zeta \in R$.

REMARK 2.3. If both (d) and (e) hold with different $q$ and $q^{\prime}$, then they are also satisfied with the same $q^{\prime \prime}=\max \left\{q, q^{\prime}\right\} \in(0,1)$. It is sufficient to prove this remark only for (d), the case (e) being obvious. We note first that the inequality in (d) holds also for every continuous function $h: \boldsymbol{R} \rightarrow \boldsymbol{R}$ such that $h(t) \geq q^{\prime \prime} M \geq q M$. Then replacing $q$ by the $q^{\prime \prime} \geq q$ in the same inequality, we will decrease the right-hand side, while the left hand side of the expression will be increased.

Now we are in a position to state the main theorems of the paper.

THEOREM 2.4. Assume (1.3) and the hypotheses (a), (b), (c), (f). Then, for every solution $x:[\tau-h, \infty) \rightarrow \boldsymbol{R}$ of (2.2) one has

$$
|x(t)| \leq \max _{s \in[\tau-h, \tau+2 h]}|x(s)| \text { for all } t \geq \tau
$$


COROLlaRY 2.5. Under the conditions of Theorem 2.4, the zero solution of (2.2) is uniformly stable and

$$
\left\|x_{t}\right\|_{C} \leq \exp \left(3 b h e^{-1}\right)\left\|x_{s}\right\|_{C}, \quad t \geq s .
$$

Replacing (c), (f) by stronger assumptions (d), (e), we obtain the exponential stability:

THEOREM 2.6. Assume (1.3) and the hypotheses (a), (b), (d), (e). Then, for every solution $x:[\tau-h, \infty) \rightarrow \boldsymbol{R}$ of $(2.2)$ one has

$$
|x(t)| \leq q \max _{s \in[\tau-h, \tau+3 h+\mu]}|x(s)| \text { for all } t \geq \tau+3 h+\mu .
$$

We can write (2.6) in a more usual form by introducing the constant

$$
\gamma=-\frac{\ln q}{\mu+4 h}>0
$$

COROLLARY 2.7. Under the assumptions of Theorem 2.6 the inequalities (2.4) and (2.6) are satisfied together with

$$
\left\|x_{t}\right\|_{C} \leq \frac{1}{q} \exp \left(3 b h e^{-1}\right)\left\|x_{s}\right\|_{C} \exp (-\gamma(t-s)), \quad t \geq s .
$$

Therefore, the zero solution of (2.2) is globally exponentially and uniformly stable.

Consider the important particular case when the function $g(t, x)$ is continuous and locally Lipschitz in $x$ and satisfies the inequalities

$$
0 \leq a x^{2} \leq g(t, x) x \leq c x^{2} \text { for all }(t, x) \in \boldsymbol{R}^{2}
$$

for some fixed $0<a<c$ (for example, one can think here of the case $g(t, x)=a(t, x) x$ with $a \leq a(t, x) \leq c, t \in \boldsymbol{R})$.

Notice that in this case (a), (b), (c), (d) hold in an obvious way and we only need to check either condition (e) or condition (f). A direct calculation (see Section 7 for details) shows that

where

$$
r(\zeta, M) \leq M \frac{b}{a}\left\{\left(1+\frac{b}{c}\right) e^{-c \xi+c h}-\frac{b}{c}\right\},
$$

$$
\xi=h+\frac{1}{c-a} \ln \frac{(b+c) /(c-a)}{(b+c) /(c-a)-e^{-a h}(1+c / b)^{a / c}} .
$$

Therefore, we have the following result:

COROLlARY 2.8. Assume that function $g(t, x)$ is continuous and locally Lipschitz in $x$ and satisfies inequalities (2.8) together with

$$
\begin{aligned}
& b h \leq 1 \text { or } \\
& {\left[1<b h \text { and } \frac{1}{c-a} \ln \frac{(b+c) /(c-a)-e^{-a h}(1+c / b)^{a / c}}{(b+c) /(c-a)} \leq \frac{1}{c} \ln \frac{a c+b^{2}}{b c+b^{2}}\right]}
\end{aligned}
$$

for some fixed $0<a<c$. Then the zero solution of (2.2) is uniformly stable for every $0 \leq b(t) \leq b$ and every continuous functional $f(t, \varphi)$ satisfying (1.3). Moreover, it is 
uniformly exponentially stable if the sign of the strict inequality $<$ in (2.9) is replaced by $\leq$, and the non-strict inequalities $\leq$ are replaced by $<$.

Obviously, $g(t, x)=a x$ when $a=c$. In this case (2.2) becomes

$$
x^{\prime}(t)+a x(t)+b(t) f\left(t, x_{t}\right)=0, \quad t \in \boldsymbol{R},
$$

and the inequality (2.9) takes the following "limit" form:

$$
\frac{a}{b} \exp (-a h) \geq \ln \left(\frac{1+a / b}{1+a^{2} / b^{2}}\right), \quad a>0, \quad b>0 .
$$

The following two theorems show to what extent this sufficient condition is sharp.

THEOREM 2.9. Let $a>0, b>0$. Then the zero solution of (2.10) is globally exponentially uniformly stable for every continuous functional $f(t, \varphi)$ satisfying (1.3) and for every $0 \leq b(t) \leq b$ if and only if the point $(a, b)$ belongs to the domain $\mathcal{D} \subset \boldsymbol{R}^{2}$ given by

$$
\frac{a}{b} \exp (-a h)>\ln \left(\frac{1+a / b}{1+a^{2} / b^{2}}\right), \quad a>0, \quad b>0 .
$$

In the limit case, when the point $(a, b)$ belongs to the boundary $\partial \mathcal{D}$ of the domain $\mathcal{D}$ of asymptotic stability, we still have the uniform stability:

THEOREM 2.10. Let $a>0, b>0$. Then the zero solution of (2.10) is uniformly stable for every continuous functional $f(t, \varphi)$ satisfying (1.3) and for every $0 \leq b(t) \leq b$ if and only if the point $(a, b)$ belongs to the domain $\mathcal{D} \cup \partial \mathcal{D}$ given by (2.11). Moreover, every solution $x:[\tau-h,+\infty) \rightarrow \boldsymbol{R}$ of (2.10) satisfies inequality (2.4).

The sufficiency part of these two theorems is a consequence of Corollary 2.8, while the necessity will be proved in Section 4.

REMARK 2.11. Considering the case of general (not necessarily positive) $b(t)$ and applying the Halanay inequality (see Section 3), we will also show that the necessary and sufficient condition for the zero solution of (2.10) to be globally exponentially uniformly stable [resp. uniformly stable] for every continuous functional $f(t, \varphi)$ satisfying $(1.3)$ and for every $|b(t)| \leq b$ is $|b|<a$ [resp. $|b| \leq a$ ].

Theorems 2.9 and 2.10 give an immediate extension of the $3 / 2$-stability criterion by Yorke [24]. In fact, by indicating the exact stability domain we obtain a family of the $3 / 2$ type stability conditions. If $a>0$, the corresponding exact upper bound $b_{a}$ for $\|b(t)\|_{\infty}$ can be found from (2.12). Moreover, the Yorke theorem corresponds to the limit case $b_{0}=3 / 2$. Indeed, the inequality $0<b<3 / 2$ is the limit form of (2.12) as $a \rightarrow 0+$, so that the closure of $\mathcal{D}$ intersected with the axis $a=0$ gives the Yorke stability interval [0,3/2].

Finally, we note that the Grossman report [5] contains a proof of the sufficiency of (2.11) for the uniform stability as well as the corresponding result for the uniform asymptotic stability in (2.10). The result was partially announced in [8], however, it was never published and remained hardly accessible and largely unknown. The sharp nature of the condition (2.11) 
was not established. This exactness is a new and essential aspect in the statements of Theorems 2.9 and 2.10. Grossman also constructed an example showing that in the case $b h>1$, (2.2) can lose its stability even under the assumptions (a), (b), (c) and (1.3). Thus, with some reservations, we can say that the conditions (e) and (f) are necessary to ensure the stability.

3. Halanay inequalities. The inequalities (1.3) suggest that the scalar equation with maxima (1.7), or the Halanay inequality (1.4), can be considered as the comparison systems for (2.10).

We begin this section by recalling the original result of Halanay (see, for instance [7, p. 378] or [3, Lemma 1.4.6]).

Proposition 3.1. Let $x:[\mu-h, \infty) \rightarrow[0, \infty)$ be a continuous function such that (1.4) is satisfied for $t \geq \mu$. If $a \geq-b>0$, then

$$
x(t) \leq\left[\max x_{\mu}\right] \exp (\gamma(t-\mu)) \text { for } t \geq \mu,
$$

where $\gamma \leq 0$ is the largest real root of the equation $\lambda+a=|b| \exp (-\lambda h)$. Moreover, $\gamma=0$ only if $a=-b$.

For (2.10), the Halanay Lemma applies even when $f\left(t, x_{t}\right)$ satisfies only the sublinearity condition (1.2), which is considerably weaker than (1.3). Here, a direct application of Proposition 3.1 gives:

COROllary 3.2. Suppose that the continuous functional $f$ satisfies (1.2) and

$$
\underset{t \in \boldsymbol{R}}{\operatorname{ess} \sup }|b(t)|=b \leq a .
$$

Then every solution $x(t)$ of (2.10) satisfies

$$
|x(t)| \leq\left[\max \left|x_{\mu}\right|\right] \exp (\gamma(t-\mu)), \quad t \geq \mu,
$$

where $\gamma \leq 0$ is the largest real root of the equation $\lambda+a=b \exp (-\lambda h)$. Note that $\gamma=0$ only if $a=b$.

Various generalizations of the Halanay Lemma to systems of vector inequalities can be found in $[3,13,14]$. Below we present a version of this result from ([14, Theorem 2.1]) for a two-dimensional case:

Proposition 3.3. Suppose functions $u, v:[\mu-h,+\infty) \rightarrow \boldsymbol{R}^{+}$satisfy the following system of inequalities:

$$
\begin{aligned}
& u(t) \leq e^{-M(t-\mu)} u(\mu)+\int_{\mu}^{t} e^{-M(t-s)}\left(B \max u_{s}+C w(s)+D \max w_{s}\right) d s, \\
& w(t) \leq F u(t)+G \max u_{t}+H \max w_{t}, \quad t \geq \mu .
\end{aligned}
$$

where all the coefficients are positive and $H<1$. Then there exists $\delta<0$ such that

$$
u(t) \leq\left[\max u_{\mu}\right] \exp (\delta(t-\mu)) ; \quad w(t) \leq\left[\max w_{\mu}\right] \exp (\delta(t-\mu)), \quad t \geq \mu
$$

if and only if $\Psi=-M+B+(C+D)(F+G)(1-H)^{-1}<0$. 
REMARK 3.4. The best value of $\delta$ above is equal to $\max \{\ln (H) / h, \lambda\}<0$, where $\lambda$ is the maximal real root of the characteristic equation

$$
\lambda+M-B e^{-\lambda h}-\frac{\left(C+D e^{-\lambda h}\right)\left(F+G e^{-\lambda h}\right)}{1-H e^{-\lambda h}}=0 .
$$

In order to apply Proposition 3.3 to equation (2.10) the sublinearity condition (1.2) alone is not sufficient, so we will use the additional assumption (1.5). The Yorke condition (1.3) is also sufficient for our purposes here, since it implies (1.5) together with (1.2). However, the inverse implication is not valid at all (e.g., check all relations with $f(\varphi)=\min \left\{\|\varphi\|_{C}, \varphi(0)+\right.$ $|\varphi(-h / 2)-\varphi(-h)|\})$.

Let us illustrate how our idea applies to (1.1):

THEOREM 3.5. Suppose that the continuous functional $f$ satisfies (1.2) and (1.5). Then either of the following two conditions

i) $(|a|+|b|) h<1,-b<a<0$,

ii) $0<b h<1, a \geq 0$,

implies the existence of $\gamma<0$ such that every solution $x:[\mu-2 h, \infty) \rightarrow \boldsymbol{R}$ to (1.1) satisfies (3.1). (Moreover, this $\gamma$ can be found explicitly in the way indicated by Remark 3.4).

PROOF. Let $x:[\mu-2 h, \infty) \rightarrow \boldsymbol{R}$ be a solution of (1.1). Then for every parameter $\alpha \in \boldsymbol{R}$, we have

$$
x^{\prime}(t)=-a x(t)-b f\left(t, x_{t}\right)=-(a-\alpha) x(t)-(b+\alpha) f\left(t, x_{t}\right)+\alpha\left(f\left(t, x_{t}\right)-x(t)\right) .
$$

Using the variation of parameters formula, we obtain

$$
x(t)=e^{-(a-\alpha)(t-\mu)} x(\mu)+\int_{\mu}^{t} e^{-(a-\alpha)(t-s)}\left[-(b+\alpha) f\left(s, x_{s}\right)+\alpha\left(f\left(s, x_{s}\right)-x(s)\right)\right] d s
$$

Now, in view of (1.2) and (1.5),

$$
\begin{aligned}
|x(t)| \leq & e^{-(a-\alpha)(t-\mu)}|x(\mu)| \\
& +\int_{\mu}^{t} e^{-(a-\alpha)(t-s)}\left[|b+\alpha| \max _{u \in[s-h, s]}|x(u)|+|\alpha| h \max _{u \in[s-h, s]}\left|x^{\prime}(u)\right|\right] d s .
\end{aligned}
$$

Introducing one more parameter $\beta$ by

$$
x^{\prime}(t)=-(a-\beta) x(t)-(b+\beta) f\left(t, x_{t}\right)+\beta\left(f\left(t, x_{t}\right)-x(t)\right),
$$

we have

$$
\left|x^{\prime}(t)\right| \leq|a-\beta||x(t)|+|b+\beta| \max _{s \in[t-h, t]}|x(s)|+|\beta| h \max _{s \in[t-h, t]}\left|x^{\prime}(s)\right| .
$$

Finally, setting $u(t)=|x(t)|$ and $w(t)=\left|x^{\prime}(t)\right|$, we can apply Proposition 3.3 to the system of inequalities (3.3) and (3.4) with $M=a-\alpha, B=|b+\alpha|, C=|\alpha| h, \quad D=$ $0, F=|a-\beta|, G=|b+\beta|, H=|\beta| h$. Hence we have the exponential stability if the real 
number

$$
\Psi^{*}=\min \Psi(\alpha, \beta)=\min _{\alpha<a,|\beta| h<1}\left\{-(a-\alpha)+|b+\alpha|+\frac{|\alpha| h}{1-|\beta| h}(|a-\beta|+|b+\beta|)\right\}
$$

is negative. Therefore, it remains to calculate the above minimum value and to prove that it is less than zero if either of the hypotheses of the theorem is satisfied.

Indeed, i) implies that $h|a|<1$, and therefore

$$
\Psi^{*} \leq \Psi(-b, a)=\frac{-(a+b)(1-h(|a|+|b|))}{1-|a| h}<0 .
$$

Analogously, if $-b<0 \leq a$, then $|b| h<1$ implies

$$
\Psi^{*} \leq \Psi(-b, 0)=-(a+b)(1-|b| h)<0 .
$$

This fact proves the sufficiency of the inequality $b h<1$ for the stability in the case $-b<$ $0 \leq a$.

Notice also that we have required all constants in (3.2) to be positive. Therefore, even if the minimal negative value $\Psi^{*}$ of $\Psi$ is achieved at a point $\left(\alpha^{*}, \beta^{*}\right)$, we need to consider some other point $(\tilde{\alpha}, \tilde{\beta})$ close to $\left(\alpha^{*}, \beta^{*}\right)$ in order to ensure the mentioned positivity and then to use Proposition 3.3. For the same reason $D$ should be taken as an arbitrary small positive number. With these reservations, we apply Remark 3.4 to calculate the convergence exponent $\gamma$

At the first glance, Theorem 2.9 includes Theorem 3.5 as a partial case when $a>0$. However, the latter was proved under the assumptions (1.2) and (1.5) which are weaker than the condition (1.3). Moreover, we have also got some results for the case of $a<0$. We also particularly emphasize the simplicity in the use of the Halanay inequality.

4. Differential equations with maxima. Equation (1.7) has appeared in the sixties in the theory of automatic control and more recently it has been a subject of new studies (see, for instance, $[1,3,10,16,1718,21]$ and further references therein). In spite of its very simple form the equation can exhibit a complicated (chaotic) behavior [17].

It was proved in [1] that (1.7) with periodic $f(t), f(t)=f(t+T)$, has at least one $T$-periodic solution $q(t)$ for every $a+b \neq 0$. It is easy to check that Theorems 2.9 and 3.5 and Corollary 3.2 can be applied to derive conditions for the global exponential attractivity of this solution. In this way, for nonnegative $a$, we completely solve the stability problem for (1.7) introduced earlier in several papers (see, e.g., references in $[1,16])$ :

THEOREM 4.1. Assume that $a \geq 0, b \neq 0$. Then (1.7) has a globally asymptotically uniformly stable $T$-periodic solution for every $T$-periodic $f(t)$ if and only if one of the following conditions is satisfied: (i) $0<-b<a$, or (ii) $b>0$ and the pair $(a, b)$ satisfies the inequality (2.12), or (iii) $a=0$ and $0<b h<3 / 2$.

Proof. The sufficiency of conditions (i) and (ii) follows from Corollary 3.2 and Theorem 2.9 respectively, and in the case (iii) we can use the Yorke theorem. Note that in the case $a<-b$ the trivial solution of (1.7) with $f \equiv 0$ is unstable (see [16] for details). If 
$a=-b$ and $f \equiv 0$, it is obvious that any constant is a periodic solution of (1.7), and the trivial solution cannot be asymptotically stable.

Finally, to prove the necessity of (ii) in the case $b>0$, we shall need the following statement from [16]:

PROPOSITION 4.2. Let $T>h$ and suppose that $x^{*}(t)$ is a T-periodic solution of $(1.7)$ with exactly two critical points $t_{\max }, t_{\min }$ over the period. Set $x^{*}(\tau(t))=\max _{s \in[t-h, t]} x^{*}(s)$. If $x^{*}(t)$ has a nondegenerate maximum at the corresponding point $t_{\max }$ (that is $x^{* \prime \prime}\left(t_{\max }\right) \neq$ $0)$, then the Poincaré map for the linear T-periodic variational equation $v^{\prime}(t)+a v(t)+$ $b v(\tau(t))=0$ along $x^{*}(t)$ has a unique non-zero characteristic multiplier $\chi$ and $x^{*}(t)$ is an exponentially stable (unstable) solution if and only if $|\chi|<1(|\chi|>1)$.

Notice that the condition $x^{*}(\tau(t))=\max _{s \in[t-h, t]} x^{*}(s)$ determines $T$-periodic function $\tau(t)$ in a unique way except only one point over period (denoted as $v$ below). Obviously, this fact does not affect our construction. Next, in order to see why the variational equation has such a form, one has to linearize (1.7) along $x^{*}(t)$ (or differentiate max functional at points $x_{t}^{*} \in C^{1}$, for more details see [16]). Without loss of generality we can assume that $t_{\max }=0, T>t_{\min }=\kappa>0$. Thus necessarily there exists a point $v \in(\kappa, T)$ such that $x^{*}(v)=x^{*}(v-h)$. Accordingly, the variational equation along $x^{*}(t)$ assumes the form

$$
v^{\prime}(t)=\left\{\begin{array}{l}
-a v(t)-b v(0), \quad 0 \leq t<h ; \\
-a v(t)-b v(t-h), \quad h \leq t<v ; \\
-(a+b) v(t), \quad v \leq t<T .
\end{array}\right.
$$

The explicit form of this equation allows us to calculate the characteristic multiplier $\chi$. For example, if $a=0$ and $v \leq 2 h$, we can find that $\chi=\left(1-b v+b^{2}(v-h)^{2} / 2\right) \exp (-b(T-v))$. If $a \neq 0$ and $v \leq 2 h$, then

$$
\chi=\exp (-(a+b)(T-v))\left\{\frac{b^{2}}{a^{2}}+\left(1+\frac{b}{a}\right) e^{-a(v-h)}\left[e^{-a h}-\frac{b}{a}+b h-v b\right]\right\} .
$$

Finally, to prove the sharp nature of condition (ii) of Theorem 4.1 we will use the following trick. Suppose that, for given $(a, b)$, we can find a $T$-periodic smooth function $q(t)$ such that it is an unstable solution of (1.7) for some perturbation $f_{q}(t)$. Then the functional differential equation

$$
v^{\prime}(t)=-a v(t)-b f\left(t, v_{t}\right)
$$

with $f\left(t, v_{t}\right)=\left[\max _{s \in[t-h, t]}(v(s)+q(s))-\max _{s \in[t-h, t]} q(s)\right]$ has the unstable zero solution, while $f(t, \varphi)$ satisfies $(1.3)$. Therefore the pair $(a, b)$ cannot belong to the stability domain $\mathcal{D}$ indicated in Theorem 2.10. Proposition 4.2 gives us a possibility to construct such unstable solutions, firstly by choosing $a>0, b>0$ to have $|\chi|>1$ and then by defining $T$-periodic perturbation as $f_{q}(t)=q^{\prime}(t)+a q(t)+b \max q_{t}$. Hence, the parameter values $(0, b)$ such that

$$
\zeta=\sup _{h \leq \nu \leq 2 h, v<T}\left|\left(1-b v+b^{2}(v-h)^{2} / 2\right) \exp (-b(T-v))\right|>1
$$


should be excluded from the stability domain $\mathcal{D}$. Since we can manage the form of the solution $q(t)$ appropriately (fortunately, we can control its shape and therefore numbers $v$ and $T$ in the formula for $\chi$ ), we find that

$$
\zeta=\sup _{h \leq \nu \leq 2 h}\left|\left(1-b v+b^{2}(v-h)^{2} / 2\right)\right|=\sup _{0 \leq x \leq b h}\left|\left(1-b h-x+x^{2} / 2\right)\right| .
$$

Analogously, if $a>0$, then

$$
\zeta=\zeta(a, b)=\sup _{0 \leq z \leq h}\left|\frac{b^{2}}{a^{2}}+\left(1+\frac{b}{a}\right) e^{-a z}\left[e^{-a h}-\frac{b}{a}-b z\right]\right|,
$$

and the corresponding pair $(a, b)$ should be excluded from the stability domain $\mathcal{D}$ if $\zeta(a, b)>$ 1.

In the case of $a=0$, it can be verified by a direct calculation that $\zeta<1$ if and only if $b h<3 / 2$. The case $a>0$ is slightly more complicated. To study it, we need to examine properties of the function

$$
\psi(z)=\frac{b^{2}}{a^{2}}+\left(1+\frac{b}{a}\right) e^{-a z}\left[e^{-a h}-\frac{b}{a}-b z\right], \quad z \in[0, h] .
$$

In the case of instability, as we have seen, necessarily one has $b>a$ (by Corollary 3.2) and $\mu=\exp (-a h) b^{-1}<h$ (since, by Corollary 2.8 , we have $b h>1>\exp (-a h)$, once the equation is unstable). Therefore $\psi(0)<1$ and $\psi(z) \rightarrow b / a>1$ as $z \rightarrow+\infty$. Furthermore, $\psi(z)$ has only one critical point $t_{c}=\mu \in(0, h)$, where it reaches its minimal value. The mentioned properties of $\psi(z)$ imply that

$$
\zeta=\max \left\{\left|\frac{b^{2}}{a^{2}}-\left(1+\frac{b}{a}\right) e^{-a \mu} \frac{b}{a}\right|,\left|\frac{b^{2}}{a^{2}}+\left(1+\frac{b}{a}\right) e^{-a h}\left[e^{-a h}-\frac{b}{a}-b h\right]\right|\right\} .
$$

Therefore, the boundary of the domain $\mathcal{E}=\{(a, b) \mid a>0, b>0$, and $\zeta(a, b)>1\}$ in $\boldsymbol{R}_{+}^{2}$ is contained in the union $\Gamma_{1} \cup \Gamma_{2}$ of the two curves $\Gamma_{1}, \Gamma_{2}$ given respectively by

$$
\frac{b^{2}}{a^{2}}-\left(1+\frac{b}{a}\right) e^{-a \mu} \frac{b}{a}=-1\left(\text { equivalently, by } \frac{a}{b} \exp (-a h)=\ln \left(\frac{1+a / b}{1+a^{2} / b^{2}}\right)\right)
$$

and

$$
\frac{b^{2}}{a^{2}}+\left(1+\frac{b}{a}\right) e^{-a h}\left[e^{-a h}-\frac{b}{a}-b h\right]=1\left(\text { equivalently, by } b=\frac{2 a \sinh (a h)}{\exp (a h)-1-a h}\right) .
$$

Since the domain $\mathcal{D}$ of the asymptotic stability found in Theorem 2.9 cannot contain any part of $\Gamma_{1}$ or $\Gamma_{2}$ inside, and it is also bounded by $\Gamma_{1}$, we conclude that $\zeta>1$ if $(a, b) \in \Gamma_{2}$, and the boundary of $\mathcal{E}$ in $\boldsymbol{R}_{+}^{2}$ is precisely $\Gamma_{1}$.

REMARK 4.3. Clearly, the exact nature of the conditions given in Theorems 2.9 and 2.10 follows immediately from this result.

REMARK 4.4. It is rather surprising that using only test functions of very special form given in Proposition 4.2, we can derive the exact stability domain for (1.7) when $a \geq 0$. We can do the same in the case $a<0$, indicating the "upper boundary" or "eventual stability 
domain" $\mathcal{E}$ for $\mathcal{D}$. Clearly, we can expect that $\mathcal{E}=\mathcal{D}$. In other words, our test functions are "dense" to detect the exact stability conditions (however, in the latter case it looks like we need to consider $v>2 h$ ).

\section{Proof of Theorem 2.4 and Corollary 2.5.}

Proof OF Theorem 2.4. Assume that, on the contrary, (2.4) does not hold. Then there is a solution $x_{t}(\varphi)$ of some initial value problem $x_{\tau}=\varphi$ such that for some $\tau_{*}>\tau+2 h$ we have

$$
\left\|x_{\tau_{*}}(\varphi)\right\|_{C}>\delta=\max _{s \in[\tau, \tau+2 h]}\left\|x_{s}(\varphi)\right\|_{C} .
$$

Set $Q=\left\{s>\tau+2 h \mid\left\|x_{s}(\varphi)\right\|_{C}>\delta\right\}$ and consider $\tau_{1}=\inf Q \geq \tau+2 h$ (so that $\left\|x_{\tau_{1}}(\varphi)\right\|_{C}=$ $\delta)$.

Let $x(\cdot)=x(\cdot, \varphi):[\tau-h,+\infty) \rightarrow \boldsymbol{R}$ be an "ordinary" solution defined as $x(t+s, \varphi)=$ $x_{t}(\varphi)(s), s \in[-h, 0], t \geq \tau$. We claim that necessarily there exists an interval $\Delta=(\alpha, \beta) \ni$ $\tau_{1}$ with $\beta \in \boldsymbol{R} \cup\{+\infty\}$ and $\tau+h \leq \tau_{1}-h \leq \alpha \leq \tau_{1}$ such that $x(\alpha)=x(\beta)=0$ and $x(t) \neq 0$ for all $t \in \Delta$.

Indeed, suppose that $|x(t)|>0$ for all $t \in\left[\tau_{1}-h, \tau_{1}\right]$. If, for example, $x(t)>0$, then by (1.3) we have $f\left(\sigma, x_{\sigma}\right) \geq 0$ almost everywhere (a.e.) in some neighborhood $U$ of $\tau_{1}$. This implies the inequality $x^{\prime}(\sigma)<0$ for almost all $\sigma \in U$, contradicting the definition of $\tau_{1}$. Hence such point $\alpha$ exists. If the graph of $x(t)$ intersects the zero level for some $t>\alpha$, then we can choose $\beta$ as the first zero. If $x(t)>0$ for all $t>\alpha$, then we have $f\left(t, x_{t}\right) \geq 0$, and therefore $x^{\prime}(t)<0$ for all $t \geq \alpha+h$. Thus $x(t)$ decreases monotonically towards some nonnegative real number $c$. In fact, $c$ cannot be positive, since integrating (2.2) between $\alpha+h$ and $t$ we derive, by using the assumption (c), a contradiction:

$$
\begin{aligned}
x(t)-x(\alpha+h) & =-\int_{\alpha+h}^{t}\left[g(s, x(s))+b(s) f\left(s, x_{s}\right)\right] d s \\
& \leq-\int_{\alpha+h}^{t}[g(s, x(s))+b(s) c] d s \rightarrow-\infty \quad \text { as } t \rightarrow+\infty .
\end{aligned}
$$

Hence in this case $x(+\infty)=0$ and we can choose $\beta=+\infty$. The case of negative $x(t)$ can be treated similarly. In the sequel, we will assume that $x(t)$ is positive at $\tau_{1}$. In the opposite case, we can define the new variable $y(t)=-x(t)$, and then it is sufficient to notice that all the previous assumptions are still valid for the functional differential equation

$$
x^{\prime}(t)+g_{1}(t, x(t))+b(t) f_{1}\left(t, x_{t}\right)=0
$$

with $g_{1}(t, x)=-g(t,-x)$ and $f_{1}(t, x)=-f(t,-x)$.

Let $M=\max _{t \in \Delta} x(t)=x(\xi)>0$, where $\xi$ is the smallest real number having this property. If, additionally, $\min x_{\xi} \geq 0$, then, by continuity of $x(t)$ and again by (1.3), we obtain that $x^{\prime}(t)<0$ in some two-sided neighborhood of $\xi$, a contradiction. Hence $\min _{s \in[\xi-h, \xi]} x(s)<$ 0 , and therefore there exists an interval $\Delta^{*}=\left(\alpha^{*}, \beta^{*}\right)$ with $\xi-h<\beta^{*} \leq \alpha$ and such that $x(t)<0$ for all $t \in \Delta^{*}$ and $x\left(\alpha^{*}\right)=x\left(\beta^{*}\right)=0$ or $\alpha^{*}=\tau-h$. 
Next, we notice that on the interval $[\alpha, \xi], x(t)$ is a solution of the following initial value problem:

$$
x^{\prime}(t)=-g(t, x(t))+h_{1}(t), \quad x(\alpha)=0,
$$

where $h_{1}(t)=-b(t) f\left(t, x_{t}\right)$. Considering now the solution $y(t, \alpha, M)$ of the comparison equation

$$
y^{\prime}(t)=-g(t, y(t))+b M, \quad y(\alpha)=0, \quad t \in[\alpha, \xi],
$$

with $b M \geq b(t)\left(-\min x_{t}\right) \geq h_{1}(t)=-b(t) f\left(t, x_{t}\right)$, we can conclude (see, e.g., comparison Theorem 1.10.2 in [12, p. 43] established for the scalar Carathéodory equations), that

$$
x(t) \leq y(t) \leq \lambda(M)=\max _{\alpha \in \boldsymbol{R}, s \in[\alpha, \alpha+h]} y(s, \alpha, M) .
$$

Evaluating the last inequality at the point $t=\xi$, we have $0<M \leq \lambda(M)$, and therefore the inequality $\sup _{z \neq 0} z^{-1} \lambda(z)<1$ implies (2.4). Also, since $\lambda(M)<b M h$, we conclude that $b h \leq 1$ is sufficient to ensure (2.4). Hence, in the sequel we will consider only the case $b h>1$

In the next stage of the proof, given $\zeta \in \boldsymbol{R}$, we consider the initial value problem (5.2) $v^{\prime}(t)=-g(t, v(t))-b v(t-h), \quad t \geq \zeta, \quad v(s)=-M \quad$ for any $s \in[\zeta-h, \zeta]$.

Obviously, the solution of (5.2) strictly increases until its possible intersection with the real axis at some point $\zeta+\Delta \zeta, \Delta \zeta>0$. We claim that $\Delta \zeta<h$. Indeed, using the method of steps on the first interval $[\zeta, \zeta+h]$, we have

$$
v^{\prime}(t)=-g(t, v(t))+b M, \quad v(\zeta)=-M .
$$

Therefore, since $b h>1$,

$$
v(\zeta+h) \geq v(\zeta)+b M h>0, \quad v^{\prime}(\zeta+\Delta \zeta)>0,
$$

which proves our claim. This last observation shows that, for $\alpha$ given above, we can find $\zeta$ such that $\alpha=\zeta+\Delta \zeta$. Or, in other words, there exists $\zeta<\alpha$ such that the solution of (5.2) is negative and nondecreasing on the interval $\Sigma=[\zeta-h, \alpha)$, and $v(\alpha)=0$. We claim that $x(t)>v(t)$ for $t \in \Sigma$. This inequality holds trivially on the interval $[\zeta-h, \zeta]$, since $|x(t)|<M$ for all $t \in[\tau, \xi)$. Suppose now that $x(m)=v(m)$ at some point $m \in(\zeta, \alpha) \subset$ $(\zeta, \zeta+h)$ and $x(t)>v(t)$ when $t \in[\zeta-h, m)$. Next, by comparing solutions of the initial value problems

$$
\begin{gathered}
v^{\prime}(t)=-g(t, v(t))+d_{2}(t), \quad v(m)=x(m), \quad d_{2}(t)=-b v(t-h)(=b M), \quad t \geq m ; \\
x^{\prime}(t)=-g(t, x(t))+d_{1}(t), \quad x(m)=v(m), \quad d_{1}(t)=-b(t) f\left(t, x_{t}\right)<d_{2}(t), \quad t \geq m,
\end{gathered}
$$

we obtain, again by using the comparison results (see also [20, Theorem 5.III]), that $x(t)<$ $v(t)$ when $t \in(m, \alpha]$. Finally, $x(\alpha)<v(\alpha)=0$, a contradiction.

Now, considering extensions of solutions of equations (2.2) and (5.2) (or, which is the same, (2.3)) to the interval $(\alpha, \alpha+h)$ in the form of the Cauchy problems

$$
\begin{gathered}
v^{\prime}(t)=-g(t, v(t))+d_{2}(t), \quad v(\alpha)=0, \quad d_{2}(t)=-b v(t-h), \quad t \geq \alpha ; \\
x^{\prime}(t)=-g(t, x(t))+d_{1}(t), \quad x(\alpha)=0, \quad d_{1}(t)=-b(t) f\left(t, x_{t}\right) \leq d_{2}(t), \quad t \geq \alpha,
\end{gathered}
$$


we conclude that $x(t)<v(t)$ for $t \in I=(\alpha, \beta) \cap(\alpha, \alpha+h)$ (notice also that $d_{2}(t)>d_{1}(t)$ for small $|t-\alpha|)$.

Hence $M$ is less than the maximal value $r(\zeta, M)$ of the solution $v(t)$ considered on the interval $I$. This contradicts to the assumption (f).

Proof of Corollary 2.5. Given $s \in \boldsymbol{R}$, consider solution $x(t)$ on the interval $[s-h, s+2 h]$. Let $\zeta \in[s, s+2 h]$ be a point of local maximum such that $|x(\zeta)|>M=\left\|x_{s}\right\|_{C}$. Proceeding as in the proof of Theorem 2.4, we claim the existence of some $\alpha \in[\zeta-h, \zeta]$, and $\beta>\alpha$ such that: i) $|x(t)|>0$ for all $t \in \Pi=(\alpha, \beta)$; ii) $x(\alpha)=0$; and iii) either $x(\beta)=0$ or $\beta=s+2 h$. Moreover, without loosing the generality, we can assume that $N=\max _{t \in \Pi} x(t)=x(\alpha+\delta)>0$ (so that $\left.N>M_{1}=\max _{t \leq \alpha}|x(t)| \geq M\right)$.

Now, considering (2.2) on the interval $(\alpha, \alpha+\delta)$ and again applying the comparison method, we obtain that $M_{1} b \delta \geq N>M_{1}$, and therefore $b \delta>1$. Hence, on the interval $[s, s+2 h]$ there exists only a finite number of points like $\alpha+\delta$. Let us numerate them in the increasing order as $\alpha_{i}+\delta_{i}, 1 \leq i \leq k$, and introduce the notation $\left|x\left(\alpha_{i}+\delta_{i}\right)\right|=N_{i}$. Obviously $\sum \delta_{i}<3 h$, and we complete the proof of the corollary by noting that

$$
\begin{gathered}
\max _{t \in[s, s+2 h]}|x(t)|=\max _{i=1, \ldots, k} N_{i} \leq M \prod\left(b \delta_{i}\right) \leq M\left(\frac{b \sum \delta_{i}}{k}\right)^{k} \\
\leq M\left(\frac{3 b h}{k}\right)^{k} \leq M \exp \left(3 e^{-1} b h\right) .
\end{gathered}
$$

6. Proof of Theorem 2.6 and Corollary 2.7. The idea of proof of Theorem 2.6 is close to that of Theorem 2.4. However, it contains a significant number of essentially different details. Rather than to refer the reader to the proof of Theorem 2.4 for similar details, we think it is more appropriate and convenient to present the proof in complete details in this Section.

Proof OF THEOREM 2.6. Assume that, on the contrary, (2.6) does not hold. Then there is a solution $x_{t}(\varphi)$ of some initial value problem $x_{\tau}=\varphi$ such that at some point $\tau_{*} \geq$ $\tau+3 h+\mu$ we obtain

$$
\left\|x_{\tau_{*}}(\varphi)\right\|_{C}>q M=q \max _{s \in[\tau, \tau+3 h+\mu]}\left\|x_{S}(\varphi)\right\|_{C} .
$$

Set $Q=\left\{s>\tau+3 h+\mu \mid\left\|x_{s}(\varphi)\right\|_{C}>q M\right\}$ and consider $v=\inf Q \geq \tau+3 h+\mu$ (so that $\left\|x_{v}(\varphi)\right\|_{C}=q M$ or $\left.v=\tau+3 h+\mu,\left\|x_{v}(\varphi)\right\|_{C}>q M\right)$.

Let $x(\cdot)=x(\cdot, \varphi):[\tau-h,+\infty) \rightarrow \boldsymbol{R}$ be an "ordinary" solution defined as $x(t+s, \varphi)=$ $x_{t}(\varphi)(s), s \in[-h, 0], t \geq \tau$. Note that there exists a point $\tau_{0} \in T^{*}=[v-\mu-h, v] \subset$ $[\tau+2 h, \nu]$ such that $\left|x\left(\tau_{0}\right)\right|<q M$. Indeed, if, for example, $x(t) \geq q M$ for all $t \in T^{*}$ then, by (d),

$$
\begin{aligned}
-x(v)+x(v-\mu) & =\int_{v-\mu}^{v}\left(g(s, x(s))+b(s) f\left(s, x_{s}\right)\right) d s \\
& \geq \int_{v-\mu}^{v}\left(g(s, x(s))+b(s) \min x_{s}\right) d s>M(1-q) .
\end{aligned}
$$


Finally, $x(v-\mu)>M(1-q)+x(v) \geq M$, a contradiction.

Hence, we deduce the existence of $\tau_{1} \geq \tau+2 h$ such that: (i) $\left\|x_{t}(\varphi)\right\|_{C} \leq M$ for all $t \in\left[\tau, \tau_{1}\right]$; (ii) $\left\|x_{\tau_{1}}(\varphi)\right\|_{C}=M q$; and (iii) $\left\|x_{t}(\varphi)\right\|_{C}>M q$ in some right neighborhood of $\tau_{1}$.

Now, we claim that necessarily there exists an interval $\Delta=(\alpha, \beta) \ni \tau_{1}$ with $\beta \in$ $\boldsymbol{R} \cup\{+\infty\}$ and $\tau+h \leq \tau_{1}-h \leq \alpha \leq \tau_{1}$ and such that $x(\alpha)=x(\beta)=0$ and $x(t) \neq 0$ for all $t \in \Delta$.

Indeed, suppose that $|x(t)|>0$ for all $t \in\left[\tau_{1}-h, \tau_{1}\right]$. If, for example, $x\left(\tau_{1}\right)>0$, then by (1.3) we have $f\left(\sigma, x_{\sigma}\right)>0$ in some open interval containing $\tau_{1}$. Therefore $x^{\prime}(\sigma)<0$ for $\sigma$ close to $\tau_{1}$, which contradicts the property (iii) of $\tau_{1}$. Hence such point $\alpha$ exists. If the graph of solution $x(t)$ intersects the zero level for some $t>\alpha$, then we can take $\beta$ as the zero. If $x(t)>0$ for all $t>\alpha$, then we have $f\left(t, x_{t}\right) \geq 0$, and therefore $x^{\prime}(t)<0$ for all $t \geq \alpha+h$. Thus, $x(t)$ decreases monotonically towards some nonnegative real number $c$. In fact, $c$ cannot be positive, since integrating (2.2) between $\alpha+h$ and $t$, we get, by the assumption (d), the contradiction (5.1). Hence in this case $x(+\infty)=0$, and we can take $\beta=+\infty$. The case of negative $x(t)$ is treated similarly. In the sequel, we will assume that $x(t)$ is positive at $\tau_{1}$. In the opposite case, we can define new variable $y(t)=-x(t)$ and apply the same arguments to the functional differential equation

$$
x^{\prime}(t)+g_{1}(t, x(t))+b(t) f_{1}\left(t, x_{t}\right)=0
$$

with $g_{1}(t, x)=-g(t,-x)$ and $f_{1}(t, x)=-f(t,-x)$.

Hence, we have $q M<N=\max _{t \in \Delta} x(t)=x(\xi)$, where $\xi$ is the smallest real number having this property. If $\min x_{\xi} \geq 0$ then, by continuity of $x(t)$ and by (1.3), we have $x^{\prime}(\sigma)<0$ almost everywhere in some open interval containing $\tau_{1}$, a contradiction. Hence $\min _{s \in[\xi-h, \xi]} x(s)<0$, and therefore there exists an interval $\Delta^{*}=\left(\alpha^{*}, \beta^{*}\right)$ with $\xi-h<$ $\beta^{*} \leq \alpha$ such that $x(t)<0$ for all $t \in \Delta^{*}$ and $x\left(\alpha^{*}\right)=x\left(\beta^{*}\right)=0$ or $\alpha^{*}=\tau-h$.

Notice next that on the interval $[\alpha, \xi], x(t)$ is a solution of the following initial value problem:

$$
x^{\prime}(t)=-g(t, x(t))+h_{1}(t), \quad x(\alpha)=0,
$$

where $h_{1}(t)=-b(t) f\left(t, x_{t}\right)$. Considering now the solution $y(t, \alpha, M)$ of the comparison equation

$$
y^{\prime}(t)=-g(t, y(t))+b M, \quad y(\alpha)=0, \quad t \in[\alpha, \xi],
$$

with $b M \geq b(t)\left(-\min x_{t}\right) \geq h_{1}(t)=-b(t) f\left(t, x_{t}\right)$, we conclude that

$$
x(t) \leq y(t) \leq \lambda(M)=\max _{\alpha \in \boldsymbol{R}, s \in[\alpha, \alpha+h]} y(s, \alpha, M) .
$$

Evaluating the last inequality at point $t=\xi$, we have $0<M q<N \leq \lambda(M)$. Therefore, the inequality $M^{-1} \lambda(M) \leq q, M \neq 0$, implies (2.6). Also, since $\lambda(M)<b M h$, we conclude that $b h \leq q<1$ is sufficient for the stability of equation (2.2). Hence, in the sequel we will consider only the case $b h \geq 1$. 
In the next stage of the proof, given $\zeta \in \boldsymbol{R}$, we consider the initial value problem (6.1) $v^{\prime}(t)=-g(t, v(t))-b v(t-h), \quad t \geq \zeta, \quad v(s)=-M \quad$ for any $s \in[\zeta-h, \zeta]$.

Obviously, a solution of (6.1) strictly increases until its possible intersection with the zero level at some point $\zeta+\Delta \zeta, \Delta \zeta \geq 0$. We claim that $\Delta \zeta<h$. Indeed, using the method of steps we have

$$
v^{\prime}(t)=-g(t, v(t))+b M, \quad v(\zeta)=-M
$$

on the first interval $[\zeta, \zeta+h]$. Therefore, since $b h \geq 1$,

$$
v(\zeta+h)>v(\zeta)+b M h \geq 0, \quad v^{\prime}(\zeta+\Delta \zeta)>0,
$$

which proves the claim. This last observation shows that, given $\alpha$, we can find $\zeta$ such that $\alpha=\zeta+\Delta \zeta$. Or, in other words, there exists $\zeta<\alpha$ such that the solution of (6.1) is negative and increasing on the interval $\Sigma=[\zeta-h, \alpha)$, and $v(\alpha)=0$. We claim that $x(t) \geq v(t)$ for $t \in \Sigma$. This inequality holds trivially on the interval $[\zeta-h, \zeta]$ since $|x(t)| \leq M$ for all $t \in[\tau, \xi)$. Suppose now that $x(m)<v(m)$ at some point $m \in(\zeta, \alpha) \subset(\zeta, \zeta+h)$. Next, while considering solutions of the following initial value problems

$$
\begin{gathered}
v^{\prime}(t)=-g(t, v(t))+d_{2}(t), \quad v(m)>x(m), \quad d_{2}(t)=-b v(t-h)(=b M), \quad t \geq m ; \\
x^{\prime}(t)=-g(t, x(t))+d_{1}(t), \quad x(m)<v(m), \quad d_{1}(t)=-b(t) f\left(t, x_{t}\right) \leq d_{2}(t), \quad t \geq m,
\end{gathered}
$$
we get, again by the comparison results from [12, 20], that $x(t)<v(t)$ when $t \in(m, \alpha]$. Finally, $x(\alpha)<v(\alpha)=0$, a contradiction.

Now, considering prolongations of solutions of equations (2.2) and (6.1) (or, which is the same, (2.3)) on the interval $(\alpha, \alpha+h)$ in the form of the Cauchy problems

$$
\begin{gathered}
v^{\prime}(t)=-g(t, v(t))+d_{2}(t), \quad v(\alpha)=0, \quad d_{2}(t)=-b v(t-h), \quad t \geq \alpha ; \\
x^{\prime}(t)=-g(t, x(t))+d_{1}(t), \quad x(\alpha)=0, \quad d_{1}(t)=-b(t) f\left(t, x_{t}\right) \leq d_{2}(t), \quad t \geq \alpha,
\end{gathered}
$$

we conclude that $x(t) \leq v(t)$ for $t \in I=(\alpha, \beta) \cap(\alpha, \alpha+h)$.

Hence $N$ does not exceed the maximal value $r(\zeta, M)$ of the solution $v(t)$ on the interval I. Since $N>M q$, this fact contradicts to the assumption (e).

PROOF OF COROLlary 2.7. First of all, we notice that (2.6) implies the inequality

$$
\left\|x_{t}\right\|_{C} \leq \max _{u \in[s-h, s+2 h+\mu]}|x(u)| \exp (-\gamma(t-(s+2 h+\mu))), \quad t \geq s .
$$

Therefore, by Theorem 2.4 and Corollary 2.5, we conclude that

$$
\begin{aligned}
\left\|x_{t}\right\|_{C} & \leq \max _{u \in[s-h, s+2 h]}|x(u)| \exp (-\gamma(t-(s+2 h+\mu))) \\
& \leq \max _{u \in[s-h, s]}|x(u)| \exp \left(3 e^{-1} b h\right) \exp (\gamma(2 h+\mu)) \exp (-\gamma(t-s)), \quad t \geq s .
\end{aligned}
$$

To derive the final estimate it is sufficient to make use of the following inequality

$$
\exp (\gamma(2 h+\mu))=\left(\frac{1}{q}\right)^{(2 h+\mu) /(4 h+\mu)}<1 / q \text {. }
$$


7. Proof of Corollary 2.8. This section contains some calculations necessary for the proof of Corollary 2.8. Here we will use the notation from Section 2 introduced before the assumption (e). First, the comparison result implies that the solution $x:[\zeta, \alpha] \rightarrow \boldsymbol{R}$ of (2.3) is greater than or equal to the solution $y:\left[\zeta^{\prime}, \alpha\right] \rightarrow \boldsymbol{R}$ of the equation

$$
y^{\prime}(t)=-c y(t)+b M, t \geq \zeta^{\prime} \geq \zeta, \quad y(s)=-M, \quad s \leq \zeta^{\prime},
$$

where $\zeta^{\prime}$ is chosen to satisfy $y(\alpha)=0$. Since every comparison system here is autonomous, we can set $\zeta^{\prime}=0$. This gives $x(t) \geq-M$ for $t \leq 0$ and

(7.1) $x(t) \geq y(t)=M\left[\frac{b}{c}-\frac{b+c}{c} \exp (-c t)\right], \quad t \in[0, \alpha], \quad$ where $\alpha=\frac{1}{c} \ln \frac{c+b}{b}$.

Notice that $\alpha \leq h$ because of $b h \geq 1$. Next, it is easy to see that the solution $x:[\alpha, h] \rightarrow$ $\boldsymbol{R}$ does not exceed the solution $z:[\alpha, h] \rightarrow \boldsymbol{R}$ of the initial value problem

$$
z^{\prime}(t)=-a z(t)+b M, \quad z(\alpha)=0 .
$$

Integrating this equation, we have

$$
x(h) \leq z(h)=M \frac{b}{a}\left[1-\exp \left(-a h+\frac{a}{c} \ln \frac{c+b}{b}\right)\right] .
$$

Finally, on the interval $(h, \alpha+h)$ we have $x(t) \leq w(t)$, where $w(t)$ is the solution of the initial value problem

$$
w^{\prime}(t)=-a w(t)-b y(t-h), \quad w(h)=z(h),
$$

and $y(t)$ is defined by (7.1). Integrating (7.2), we obtain

$$
w(t)=C e^{-a t}+M\left[\frac{b(c+b)}{(a-c) c} e^{-c(t-h)}-\frac{b^{2}}{c a}\right],
$$

where

$$
C=\frac{b}{a} M\left[e^{a h} \frac{c+b}{c-a}-\left(1+\frac{c}{b}\right)^{a / c}\right] .
$$

Notice that $C>0$, since it can be represented as the sum of three positive numbers:

$$
C=M \exp (a h)\left[\frac{b}{a}\left(1-\exp \left(-a h+\frac{a}{c} \ln \frac{c+b}{b}\right)\right)+\frac{b^{2}}{c a}+\frac{b(c+b)}{c(c-a)}\right] .
$$

Now, the unique critical point $\xi$ of $w(t)$ (its explicit expression is given before the statement of Corollary 2.8) can be found by solving the equation $w^{\prime}(\xi)=-a w(\xi)-b y(\xi-h)=0$. Moreover, $\xi \in(h, \alpha+h]$, since

$$
w(h)=z(h)>0, w(+\infty)<0, \quad \text { and } \quad w^{\prime}(h)=M b \exp \left(-a h+\frac{a}{c} \ln \frac{c+b}{b}\right)>0,
$$

and

$$
w^{\prime}(\alpha+h)=-a w(\alpha+h)-b y(\alpha)=-a w(\alpha+h) \leq 0
$$


(note that $w(t)$ has at least two critical points in the case $w(\alpha+h)<0, w^{\prime}(\alpha+h)>0$ ). Therefore, we have

$$
r_{0}(\zeta, M)=\max _{t \in(\alpha, \alpha+h)} x(t) \leq w(\xi)=-\frac{b}{a} y(\xi-h) .
$$

By applying the symmetry argument, we also find that

$$
r_{1}(\zeta, M) \leq-\frac{b}{a} y(\xi-h)=M \frac{b}{a}\left\{\left(1+\frac{b}{c}\right) e^{-c \xi+c h}-\frac{b}{c}\right\} .
$$

\section{REFERENCES}

[ 1 ] N. R. BAntsur And E. P. Trofimchuk, Existence and stability of the periodic and almost periodic solutions of quasilinear systems with maxima, Ukrainian Math. J. 6 (1998), 747-754.

[2] D. I. BARNEA, A method and new results for stability and instability of autonomous functional differential equations, SIAM J. Appl. Math. 17 (1969), 681-697.

[ 3 ] K. Gopalsamy, Stability and oscillations in delay differential equations of population dynamics, Mathematics and its Applications 74, Kluwer Academic Publishers, Dordrecht, 1992.

[ 4 ] K. Gopals amy and S. TrofimchuK, Almost periodic solutions of Lasota-Wazewska type delay differential equation, J. Math. Anal. Appl. 237 (1999), 106-127.

[ 5 ] S. E. Grossman, Stability and asymptotic behavior of differential-delay equations, Tech. Rep. BN 611, Institute for Fluid Dynamics and Applied Mathematics, University of Maryland, College Park, 1969.

[6] S. E. Grossman AND J. A. Yorke, Asymptotic behavior and exponential stability criteria for differential delay equations, J. Differential Equations 12 (1972), 236-255.

[ 7 ] A. HalanAy, Differential equations: Stability, oscillations, time lags, Academic Press, New York and London, 1966.

[ 8 ] A. HALANAY AND J. A. YoRKE, Some new results and problems in the theory of differential-delay equations, SIAM Rev. 13 (1971), 55-80.

[ 9 ] J. KATO, On Liapunov-Razumikhin type theorems for functional differential equations, Funkcial. Ekvac. 16 (1973), 225-239.

[10] V. KolmanOVSKII AND A. MYSHKIS, Introduction to the theory and applications of functional differential equations, Mathematics and its Applications 463, Kluwer Academic Publishers, Dordrecht, 1999.

[11] T. KRISZTIN, On stability properties for one-dimensional functional differential equations, Funkcial. Ekvac. 34 (1991), 241-256.

[12] V. Lakshmikantham and S. Leela, Differential and Integral Inequalities. Theory and Applications, Vol. I, Academic Press, New York and London, 1969.

[13] L. Li-Ming, Stability of linear neutral delay-differential systems, Bull. Austral. Math. Soc. 38 (1988), 339344.

[14] E. LiZ AND S. TROFImChuK, Existence and stability of almost periodic solutions for quasilinear delay systems and Halanay inequality, J. Math. Anal. Appl. 248 (2000), 625-644.

[15] H. Matsunaga, R. Miyazaki and T. HaRA, Global attractivity results for nonlinear delay differential equations, J. Math. Anal. Appl. 234 (1999), 77-90.

[16] M. Pinto and S. Trofimchuk, Stability and existence of multiple periodic solutions for a quasilinear differential equation with maxima, Proc. Roy. Soc. Edinburgh Sect. A 130 (2000), 1103-1118.

[17] M. Pinto, S. TRofimchuK AND E. TrofimchuK, Chaotic motions in a weakly nonlinear functional equation, Actas V Simposio Chileno de Matemática, Olmué, Chile, 1998, Actas de Resúmenes, pp. 71-73.

[18] E. Stepanov, On solvability of some boundary value problems for differential equations with "maxima", Topol. Methods Nonlinear Anal. 8 (1996), 315-326.

[19] S. TRofimchuk AND A. IVANov, Weak hyperbolicity of delay differential equations and the 3/2-type stability conditions, Funkcial. Ekvac. 43 (2000), 39-56.

[20] W. WALTER, Differential and Integral Inequalities, Springer-Verlag, Berlin, 1970. 
[21] H. K. XU AND E. Liz, Boundary value problems for differential equations with maxima, Nonlinear Stud. 3 (1996), 231-241.

[22] T. Yoneyama And J. Sugie, On the stability region of differential equations with two delays, Funkcial. Ekvac. 31 (1988), 233-240.

[23] T. Yoneyama And J. Sugie, On the stability region of scalar delay-differential equations, J. Math. Anal. Appl. 134 (1988), 408-425.

[24] J. A. YoRKE Asymptotic stability for one dimensional differential-delay equations, J. Differential Equations 7 (1970), 189-202.

DEPARTMENT OF MATHEMATICS

PenNS ylVania State University

P.O BOX PSU, LEHMAN, PA 18627

U.S.A.

E-mail address: afi1@psu.edu

Departamento de MATEMáticas

FACUlTAD DE CiENCIAS

UNIVERSIDAD DE CHILE

CASILla 653, SANTIAGO

CHILE

E-mail address: trofimch@uchile.cl
DEPARTAMENTO DE Matemática APLICADA II

E.T.S.I. TELECOMUNICACIÓN

UNIVERSIDAD DE VIGO

Campus Marcosende, 36280 Vigo

SPAIN

E-mail address: eliz@dma.uvigo.es 